Pacific Journal of Mathematics






\section{STRONGLY UNICOHERENT CONTINUA}

\section{E. BENNETT}

In this journal the author introduced the concept of strongly unicoherent continua and proved that such a continuum is aposyndetic at a point $p$ if and only if it is connected im kleinem at $p$. In the present paper we obtain some new results on strongly unicoherent continua. The main theorem states that the strongly unicoherent continuum $M$ contains a unique irreducible subcontinuum between two points $p$ and $q$ provided $M$ is both aposyndetic and semi-locally-connected at $p$.

Throughout this paper a continuum is a compact connected metric space and $M$ will denote a continuum. The continuum $M$ is unicoherent if whenever $M=A \cup B$, with $A$ and $B$ subcontinua of $M, A \cap B$ is connected. $\quad M$ is hereditarily unicoherent if every subcontinuum of $M$ is unicoherent. A continuum $M$ is said to be irreducible between a pair of points $p$ and $q$ of $M$ provided no proper subcontinuum of $M$ contains both $p$ and $q . \quad M$ is said to be irreducible if there exists two points so that $M$ is irreducible between the pair of points.

If $N$ is a subset of $M$, the interior of $N$ in $M$ will be denoted by int $N$ and the closure of $N$ in $M$ by $\bar{N}$.

For other terms not defined herein see [3] and [5].

Definition 1. A unicoherent continuum $M$ is strongly unicoherent provided that for any pair of proper subcontinua $H$ and $K$ such that $M=H \cup K$, each of $H$ and $K$ is unicoherent.

It is easily seen that this notion is a stronger form of unicoherence, but is somewhat weaker than hereditarily unicoherence. For example, a continuum which consists of a ray limiting on a circle is strongly unicoherent but fails to be hereditarily unicoherent.

In [4] Miller proved the following theorem.

THEOREM 1. If the atriodic unicoherent continuum $M$ is the sum of two proper subcontinua $H$ and $K$, then $H$ and $K$ are unicoherent, and if $N$ is a nonunicoherent subcontinuum of $M$ intersecting $H$, it is a subset of $H$.

Thus, atriodic unicoherent continua are examples of continua which are strongly unicoherent. A reasonable conjecture is that the last portion of the theorem holds for strongly unicoherent continua, but this is not the case as shown by the following example. 


\section{EXAMPLE 1.}

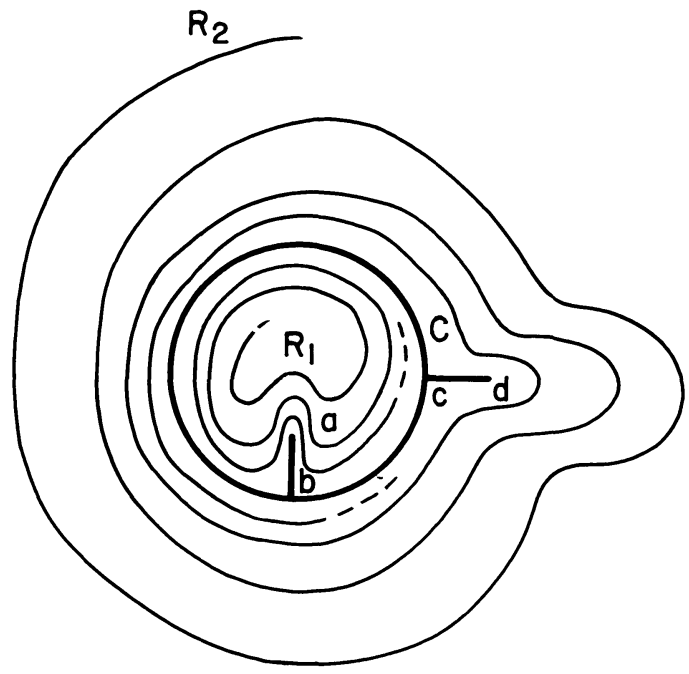

The strongly unicoherent continuum $M$ consist of a circle $C$, two arcs $[a, b]$ and $[c, d]$ which intersect $C$ at the point $b$ and point $c$ respectively, a ray $R_{1}$ which limits on $C \cup[a, b]$, and a ray $R_{2}$ which limits on $C \cup[c, d]$.

Let $H=\bar{R}_{1}$ and $K=\bar{R}_{2} \cup[a, b]$. Then $H$ and $K$ are proper subcontinua of $M$ and $M=H \cup K$. Let

$$
N=C \cup[a, b] \cup[c, d] .
$$

$N$ is non-unicoherent, $N \cap H \neq \varnothing$, but $N \not \subset H$.

Note that while $M=H \cup K$ and $K$ is unicoherent it does not follow that $K$ is strongly unicoherent as shown by the example above.

COROLlaRY 1. If $M$ is atriodic, then every unicoherent subcontinuum of $M$ is strongly unicoherent.

Next we investigate the relationship of a strongly unicoherent continuum $M$ and its nonunicoherent subcontinua.

DEFInition 2. A subcontinuum $N$ of $M$ is said to be a continuum of condensation if each point of $N$ is a limit point of $M-N$.

The next theorem is an immediate consequence of definitions 1 and 2.

THEOREM 2. If $N$ is a nonunicoherent subcontinuum of a strongly unicoherent continuum $M$ then either (i) $N$ separates $M$ or, (ii) $N$ is a continuum of condensation. 
COROLlaRY 2. If $N$ is a nonunicoherent subcontinuum of a strongly unicoherent continuum $M$ then each subcontinuum of $N$ either (i) separates $M$ or (ii) is a continuum of condensation.

Question. Is every nonunicoherent subcontinuum of a strongly unicoherent continuum $M$ a continuum of condensation?

The answer to the preceding question is in the affirmative if the continuum $M$ is also irreducible.

THEOREM 3. Suppose that $M$ is a strongly unicoherent irreducible continuum. Then every nonunicoherent subcontinuum of $M$ is a continuum of condensation in $M$.

Proof. Let $a$ and $b$ be in $M$ such that $M$ is irreducible between $a$ and $b$ and suppose $N$ is a non-unicoherent subcontinuum of $M$. According to Theorem 2, we may assume that $N$ separates $M$. It follows that $\{a, b\} \cap N=\varnothing$ and $M-N$ has exactly two components, say $A$ and $B$. Without loss of generality, assume that $a \in A$ and $b \in B$. If $\bar{A} \cap \bar{B} \neq \varnothing$ then $\bar{A} \cup \bar{B}$ is a subcontinuum of $M$ containing $\{a, b\}$. Thus, $M=\bar{A} \cup \bar{B}$ and it follows that $N$ is a continuum of condensation.

Suppose that $\bar{A} \cap \bar{B}=\varnothing$. Let $H$ and $K$ be subcontinua of $N$ such that $N=H \cup K$ and $H \cap K$ is not connected.

Assertion. $\quad \bar{A} \cap H \neq \varnothing \neq H \cap \bar{B}$. For suppose that this is not the case. Assume that $\bar{A} \cap H=\varnothing$. Then $\bar{A} \cap K \neq \varnothing$ and $\bar{A} \cup K \cup H$ is a proper subcontinuum of $M$. Since $M=(\bar{A} \cup K \cup H) \cup \bar{B}$, it follows that $\bar{A} \cup H \cup K$ is unicoherent. This implies that $(\bar{A} \cup K) \cap H=$ $H \cap K$ is connected which is a contradiction. Thus the assertion holds.

In a similar manner, it follows that $\bar{A} \cap K \neq \varnothing \neq K \cap \bar{B}$. Since $M=\bar{A} \cup H \cup \bar{B}$, then $K-H \subset \bar{A} \cup \bar{B}$. Also since $M=\bar{A} \cup K \cup \bar{B}$, it follows that $H-K \subset \bar{A} \cup \bar{B}$.

Finally we will show that $H \cap K \subset \bar{A} \cup \bar{B}$. Let $P$ and $Q$ be disjoint closed sets such that $H \cap K=P \cup Q$, let $p \in P$ and $C$ be the component of $p$ in $H-Q$. Then $\bar{C} \cap Q \neq \varnothing$, but note that $\bar{C} \cap$ (int $Q)=\varnothing$. Since $\bar{C} \cup K \cup \bar{A}$ is a proper subcontinuum of $M$ and $M=\bar{B} \cup(\bar{C} \cup K \cup \bar{A})$, then $\bar{C} \cup K \cup \bar{A}$ is unicoherent. Thus $(K \cup \bar{A}) \cap \bar{C}=(K \cap \bar{C}) \cup(\bar{C} \cap \bar{A})$ is connected. Now $(K \cap \bar{C}) \cap$ $P \neq \varnothing \neq(K \cap \bar{C}) \cap Q$ and $K \cap \bar{C} \subset P \cup Q$ which implies that $K \cap \bar{C}$ is not connected. It follows that $\bar{C} \cap \bar{A} \neq \varnothing$.

By interchanging the roles of $\bar{A}$ and $\bar{B}$ in the above argument, we have that $\bar{C} \cap \bar{B} \neq \varnothing$. Now $\bar{A} \cup \bar{C} \cup \bar{B}$ is a subcontinuum of $M$ containing $\{a, b\}$, so $M=\bar{A} \cup \bar{C} \cup \bar{B}$. Since (int $Q$ ) $\cap \bar{C}=\varnothing$, then int $Q) \subset \bar{A} \cup \bar{B}$. 
Suppose $q \in Q-$ (int $Q$ ) and $V$ is an open set containing $q$ such that $V \cap P=\varnothing$. Then $V \not \subset Q$ so there is a point $z \in V \cap$ $(M-Q)$. Since $z \notin P \cup Q$ it follows by the first portion of this proof that $z \in \bar{A} \cup \bar{B}$. Thus $q$ is a limit point of $\bar{A} \cup \bar{B}$ and hence $q \in \bar{A} \cup$ $\bar{B}$. Therefore $Q \subset \bar{A} \cup \bar{B}$.

Since the preceding argument is symmetric with respect to $P$ and $Q$, it follows that $P \subset \bar{A} \cup \bar{B}$.

Therefore $N=(H-K) \cup(K-H) \cup P \cup Q \subset \bar{A} \cup \bar{B}$ which implies that $N$ is a continuum of condensation.

The following well known characterization of hereditarily unicoherent continua was given in [4].

THEOREM 4 (Miller). In order that the continuum $M$ be hereditarily unicoherent it is necessary and sufficient that for any two points $p$ and $q$ of $M$ there is only one subcontinuum of $M$ which is irreducible between $p$ and $q$.

DEFINITION 3. A continuum $M$ is hereditarily unicoherent at the point $p$ of $M$ provided for each $q \in M$ different from $p$, there is a unique subcontinuum of $M$ which is irreducible between $p$ and $q$.

Thus if $M$ is hereditarily unicoherent at $p$ and $q \in M-\{p\}$, then the intersection of all subcontinuum of $M$ which contain $\{p, q\}$ is connected.

We shall show that strongly unicoherent continua are "hereditarily unicoherent at certain points", but first we prove the following lemma.

LeMmA 1. Let $p$ and $q$ be points of the continuum $M, I_{1}$ and $I_{2}$ be subcontinua of $M$ which are irreducible between $p$ and $q$, and $D$ be $a$ subcontinuum of $M$ containing $p$. If the continuum $D \cup I_{1} \cup I_{2}$ is unicoherent, then $I_{1} \cap M-D=I_{2} \cap M-D$.

Proof. Suppose that $D \cup I_{1} \cup I_{2}$ is unicoherent. Then $\left(D \cup I_{2}\right) \cap$ $I_{1}$ is connected and hence is a subcontinuum of $I_{1}$ containing $\{p, q\}$. Since $I_{1}$ is irreducible between $p$ and $q$, then $\left(D \cup I_{2}\right) \cap I_{1}=$ $I_{1}$. Therefore $I_{1} \subset D \cup I_{2}$ which implies that (i) $I_{1} \cap(M-D) \subset I_{2}$.

Likewise $\left(D \cup I_{1}\right) \cap I_{2}$ is subcontinuum of $I_{2}$ containing $\{p, q\}$ so $\left(D \cup I_{1}\right) \cap I_{2}=I_{2}$. Thus $I_{2} \subset D \cup I_{1}$ and it follows that (ii) $I_{2} \cap$ $(M-D) \subset I_{1}$.

The conclusion follows from (i) and (ii).

THEOREM 5. Let $M$ be a strongly unicoherent continuum and $p \in M$. If $M$ is both aposyndetic at $p$ and semi-locally-connected at $p$, then $M$ is hereditarily unicoherent at $p$.

Proof. Suppose that $M$ is aposyndetic and semi-locally-connected 
at $p, q \in M-\{p\}$, and $I_{1}$ and $I_{2}$ are subcontinua of $M$ which are irreducible between $p$ and $q$.

Since $M$ is aposyndetic and semi-locally-connected at $p$, there are subcontinua $H_{1}$ and $K_{1}$ of $M$ such that $p \in H_{1}-K_{1}, q \in K_{1}-H_{1}$, and $M=H_{1} \cup K_{1}$ (Theorem 6 of [2]).

Since $M$ is aposyndetic at $p$, according to Theorem 6 of [1], $M$ is also connected im kleinen at $p$. So there is a subcontinuum $L$ in $M-\left(H_{1} \cap K_{1}\right)$ such that $p \in$ int $L$. Since $M$ is semi-locally-connected at $p$, there is an open set $V$ such that $p \in V \subset$ (int $L$ ) and $M-V$ has a finite number of components. Let $F_{1}, \cdots, F_{n}$ be the components of $M-V$ and without loss of generality assume that $K_{1} \subset F_{1}$. Let $H_{2}=$ $L \cup\left(F_{2} \cup F_{3} \cup \cdots \cup F_{n}\right)$ and $K_{2}=F_{1}$. Then $H_{2}$ and $K_{2}$ are subcontinua such that $p \in H_{2}-K_{2}, q \in K_{2}-H_{2}$, and $M=H_{2} \cup K_{2}$.

Since $M$ is strongly unicoherent, then $K_{1} \cup I_{1} \cup I_{2}, H_{2} \cup I_{1} \cup I_{2}$, and $K_{2} \cup I_{1} \cup I_{2}$ are unicoherent. So by the preceding lemma, $I_{1} \cap$ $\left(M-K_{1}\right)=I_{2} \cap\left(M-K_{1}\right), \quad I_{1} \cap\left(M-H_{2}\right)=I_{2} \cap\left(M-H_{2}\right), \quad$ and $I_{1} \cap\left(M-K_{2}\right)=I_{2} \cap\left(M-K_{2}\right)$.

Now $H_{2} \cap K_{2} \subset L \subset M-K_{1}$ so $I_{1} \cap\left(H_{2} \cap K_{2}\right)=I_{2} \cap\left(H_{2} \cap K_{2}\right)$. So it follows that

$$
\begin{aligned}
& I_{1}=\left[I_{1} \cap\left(M-H_{2}\right)\right] \cup\left[I_{1} \cap\left(H_{2} \cap K_{2}\right)\right] \cup\left[I_{1} \cap\left(M-K_{2}\right)\right] \\
= & {\left[I_{2} \cap\left(M-H_{2}\right)\right] \cup\left[I_{2} \cap\left(H_{2} \cap K_{2}\right)\right] \cup\left[I_{2} \cap\left(M-K_{2}\right)\right]=I_{2} . }
\end{aligned}
$$

Therefore $M$ is hereditarily unicoherent at $p$.

COROLlaRY 3. If the strongly unicoherent $M$ is aposyndetic at each point, then $M$ is hereditarily unicoherent.

Proof. By Theorem 4 of [2] $M$ is semi-locally-connected at each point, so it follows from the preceding theorem that $M$ is hereditarily unicoherent.

\section{REFERENCES}

1. D. E. Bennett, Aposyndetic properties of unicoherent continua, Pacific J. Math., 37 (1971), 585-589.

2. F. B. Jones, Aposyndetic continua and certain boundary problems, Amer. J. Math., 63 (1941), $545-553$.

3. K. Kuratowski, Topologie Vol. II PWN-Academic Press, (1968) Warsaw-New York.

4. H. C. Miller, On unicoherent continua, Trans. Amer. Math. Soc., 69 (1950), 179-194.

5. G. T. Whyburn, Analytical Topology, Amer. Math. Soc. Colloq. Publications 28 (1942), Providence, R.I.

Received November 1, 1974. A portion of the research reported in this paper was done while the author was a recipient of a research fellowship at the University of Kentucky during the summer of 1974.

MURRAY STATE UNIVERSITY

AND

UNIVERSITY OF KENTUCKY 

D. E. Bennett, Strongly unicoherent continua ............................. 1

Walter R. Bloom, Sets of p-spectral synthesis ................................ 7

R. T. Bumby and D. E. Dobbs, Amitsur cohomology of quadratic extensions: Formulas and number-theoretic examples ................. 21

W. W. Comfort, Compactness-like properties for generalized weak topological sums

D. R. Dunninger and J. Locker, Monotone operators and nonlinear biharmonic boundary value problems ...

T. S. Erickson, W. S. Martindale, 3rd and J. M. Osborn, Prime nonassociative algebras

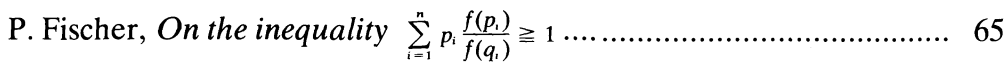

G. Fox and P. Morales, Compact subsets of a Tychonoff set ............... 75

R. Gilmer and J. F. Hoffmann, A characterization of Prüfer domains in terms of polynomials ......................................................... 81

L. C. Glaser, On tame Cantor sets in spheres having the same projection in each direction ......................................................... 87

Z. Goseki, On semigroups in which $X=X Y X=X Z X$ if and only if

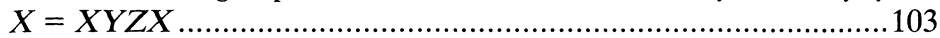

E. Grosswald, Rational valued series of exponentials and divisor

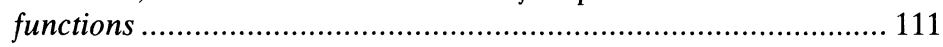

D. Handelman, Strongly semiprime rings ...................................... 115

J. N. Henry and D. C. Taylor, The $\bar{\beta}$ topology for $w^{*}$-algebras ............. 123

M. J. Hodel, Enumeration of weighted p-line arrays .......................... 141

S. K. Jain and S. Singh, Rings with quasiprojective left ideals .............. 169

S. Jeyaratnam, The diophantine equation $Y(Y+m)(Y+2 m) \times$

$$
(Y+3 m)=2 X(X+m)(X+2 m)(X+3 m) \ldots \ldots \ldots \ldots \ldots \ldots \ldots . . .183
$$

$\mathrm{R}$. Kane, On loop spaces without $p$ torsion .........................................189

Alvin J. Kay, Nonlinear integral equations and product integrals ..........203

A. S. Kechris, Countable ordinals and the analytic hierarchy, I ...........223

Ka-Sing Lau, A representation theorem for isometries of $C(X, E) \ldots \ldots . .229$

I. Madsen, On the action of the Dyer-Lashof algebra in $H_{*}(G)$..........235

R. C. Metzler, Positive linear functions, integration, and Choquet's theorem ........................................................................................ 277

A. Nobile, Some properties of the Nash blowing-up ............................297

G. E. Petersen and G. V. Welland, Plessner's theorem for Riesz conjugates 


\section{Pacific Journal of Mathematics}

\section{Vol. 60, No. $1 \quad$ September, 1975}

Donald Earl Bennett, Strongly unicoherent continua ................ 1

Walter Russell Bloom, Sets of p-spectral synthesis ................ 7

Richard Thomas Bumby and David Earl Dobbs, Amitsur cohomology of

quadratic extensions: formulas and number-theoretic examples .......

W. Wistar (William) Comfort, Compactness-like properties for generalized

weak topological sums ...................................

Dennis Robert Dunninger and John Stewart Locker, Monotone operators

and nonlinear biharmonic boundary value problems ..............

Theodore Erickson, Wallace Smith Martindale, III and J. Marshall Osborn,

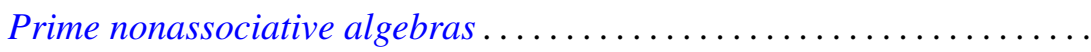

Pál Fischer, On the inequality $\sum_{i=0}^{n}\left[f\left(p_{i}\right) / f\left(q_{i}\right)\right] p_{i} \geq i \ldots \ldots \ldots \ldots \ldots$

Geoffrey Fox and Pedro Morales, Compact subsets of a Tychonoff set.......

Robert William Gilmer, Jr. and Joseph F. Hoffmann, A characterization of

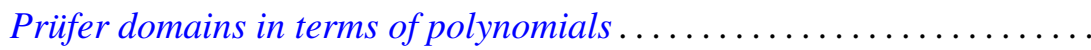

Leslie C. Glaser, On tame Cantor sets in spheres having the same projection

in each direction . ...................................

Zensiro Goseki, On semigroups in which $x=x y x=x z x$ if and only if



Emil Grosswald, Rational valued series of exponentials and divisor

functions.

David E. Handelman, Strongly semiprime rings

Jackson Neal Henry and Donald Curtis Taylor, The $\bar{\beta}$ topology for

$W^{*}$-algebras

Margaret Jones Hodel, Enumeration of weighted p-line arrays ...

Surender Kumar Jain and Surjeet Singh, Rings with quasi-projective left

ideals.

S. Jeyaratnam, The Diophantine equation

$$
Y(Y+m)(Y+2 m)(Y+3 m)=2 X(X+m)(X+2 m)(X+3 m) \ldots
$$

Richard Michael Kane, On loop spaces without $p$ torsion

Alvin John Kay, Nonlinear integral equations and product integrals ...

Alexander S. Kechris, Countable ordinals and the analytical hierarchy.

$I$.

Ka-Sing Lau, A representation theorem for isometries of $C(X, E)$

Ib Henning Madsen, On the action of the Dyer-Lashof algebra in $H_{*}(G)$

Richard C. Metzler, Positive linear functions, integration, and Choquet's

theorem.

Augusto Nobile, Some properties of the Nash blowing-up

Gerald E. Peterson and Grant Welland, Plessner's theorem for Riesz. 